\section{Disparidades en salud mental asociadas a la orientación sexual en adolescentes mexicanos}

\author{
Disparities in mental health associated with sexual \\ orientation among Mexican adolescents
}

\author{
Desigualdades em saúde mental associada com a \\ orientação sexual em adolescentes mexicanos
}

\author{
1 Departamento de Atención \\ a la Salud, Universidad \\ Autónoma Metropolitana \\ Unidad Xochimilco, México \\ DF, México. \\ Correspondencia \\ L. Ortiz-Hernández \\ Departamento de Atención \\ a la Salud, Universidad \\ Autónoma Metropolitana \\ Unidad Xochimilco. \\ Calz. del Hueso 1100, Col. \\ Villa Quietud, Del. Coyoacán, \\ México / Distrito Federal - \\ 04960, México. \\ lortiz@correo.xoc.uam.mx
}

\begin{abstract}
The aim of this study was to document disparities in mental health related to discrimination based on sexual orientation in Mexican adolescents. A representative national sample of secondary school students was analyzed. Criteria for homosexual orientation were having had a same-sex boyfriend or girlfriend and having had same-sex sexual relations. The events were: depression, low self-esteem, suicidal ideation, attempted suicide, smoking, alcohol abuse, and drug use. Teenagers with same-sex relationships or sexual relations had an increased risk of depressive symptoms, suicidal ideation, attempted suicide, and alcohol abuse. These differences were particularly related to having experienced violence in the family and in school. Despite institutional and legal progress in acknowledging the rights of the lesbian, bisexual, and gay population, health inequities persist due to discrimination based on sexual orientation.
\end{abstract}

Homophobia; Mental Health; Sexual Behavior; Violence
Luis Ortiz-Hernández 1

Reyna Guadalupe Valencia-Valero ${ }^{1}$

\section{Resumen}

El objetivo de este estudio fue documentar las disparidades en salud mental relacionadas con la discriminación por orientación sexual en adolescentes de México. Se estudió una muestra nacional representativa de estudiantes de bachillerato. Se usaron dos indicadores de orientación homosexual: haber tenido un novio del mismo sexo y haber tenido relaciones sexuales con alguien del mismo sexo. Los eventos fueron: depresión, autoestima, ideación suicida e intento de suicidio y consumo de cigarros, alcohol y otras drogas. Los adolescentes que habian tenido noviazgos o relaciones sexuales con personas de su mismo sexo tuvieron un riesgo incrementando de síntomas depresivos, ideación e intento de suicidio y consumo problemático de alcohol. Estas diferencias sobre todo se relacionaron con la experiencia de violencia en la familia y la escuela. A pesar de los avances institucionales y legales para el reconocimiento de los derechos de la población lésbica, bisexual y homosexual, persisten inequidades en salud relacionadas con la discriminación por orientación sexual.

Homofobia; Salud Mental; Conducta Sexual; Violencia 


\section{Introducción}

En el creciente reconocimiento del papel que tienen los determinantes sociales en la configuración de los perfiles de salud de las poblaciones, ha predominando la preocupación por los estragos causados por la pobreza y la estratificación socioeconómica. Al mismo tiempo otros determinantes sociales como el prejuicio y la discriminación han sido ignorados o desestimados. Por ejemplo, en el primer informe de la Comisión sobre Determinantes Sociales de la Salud de la Organización Mundial de la Salud ${ }^{1}$ sólo se hace mención a la discriminación por "razones de sexo", la discriminación hacia los niños con discapacidad y la discriminación en el empleo. Además, sólo una de sus recomendaciones está encaminada directamente a la erradicación de la discriminación por género; con lo cual se omiten el resto de formas de prejuicio y discriminación, e.g. las relacionadas con la racialización o la homofobia.

En el caso específico de la discriminación asociada con la orientación sexual, con excepciones ${ }^{2}$, la mayoría de la evidencia proviene de países de alta renta ${ }^{3,4}$. Debido a las diferencias socioeconómicas y culturales, las actitudes negativas hacia la homosexualidad son más frecuentes entre la población de países con ingresos medios o bajos. Por ello, es predecible que las disparidades en salud, asociadas con la orientación sexual, sean mayores en las sociedades no industrializadas. De acuerdo con la Encuesta Mundial de Valores (EMV) (World Values Survey. http://www.world valuesurvey.org, accedido el 09/Oct/2012), en países industrializados la proporción de personas que piensan que la homosexualidad es una conducta que nunca puede justificarse es menor que en las naciones latinoamericanas: $7,0 \%$ en Holanda; 8,4\% en Suecia; 25,9\% en Bélgica; 25,3\% en Canadá y 31,0\% en los Estados Unidos; en contraste, en Chile es de $35,1 \%$, en México de $48,3 \%$, Perú de 55,8\%, y Venezuela de $61,1 \%$. Estas actitudes prevalecientes en cada sociedad se han materializado en arreglos institucionales que apoyan o niegan los derechos de las lesbianas, bisexuales y homosexuales (LBH). Mientras que en varios países europeos el matrimonio entre personas del mismo sexo fueron aprobados desde el siglo pasado; en los países latinoamericanos este reconocimiento legal de las familias no heterosexuales ha comenzado en años recientes. Otra diferencia de México con los países industrializados es que la religiosidad es más frecuente. Además de que la jerarquía de la iglesia católica tiene una postura abiertamente homofóbica.

Considerando que la discriminación se construye en torno a las creencias sesgadas que se ha- ce de un grupo, una pregunta que surge es: ¿qué implica pertenecer a un grupo que es objeto de discriminación?, es decir, ¿ cómo experimentan el prejuicio aquellas personas que son objeto de éste? De acuerdo con Goffman 5, en los individuos que portan algún estigma existen dos etapas importantes en su socialización, la primera es cuando la persona con el estigma incorpora el punto de vista dominante con lo que adquiere las creencias sobre tal estigma y una idea general de lo que significa portarlo; en la otra fase el individuo aprende que porta un estigma y qué consecuencias puede conllevar el tenerlo. De esta forma, las personas que portan algún estigma internalizan en su auto-imagen o auto-concepto los significados negativos, los prejuicios y los estereotipos asociados con la condición que portan; lo que puede provocar que tengan actitudes y reacciones negativas hacia su propia persona. Esta situación correspondería a la homofobia internalizada que experimentan las LBH 6 .

Considerando lo anterior, el objetivo de este estudio fue documentar las disparidades en salud mental relacionadas con la discriminación por orientación sexual en adolescentes de México. Además, se evalúo si las experiencias de violencia en la escuela y la familia, así como las actitudes hacia la homosexualidad podían explicar las diferencias observadas.

\section{Material y métodos}

Se analizó la base de datos de la segunda Encuesta Nacional Sobre Exclusión, Intolerancia y Violencia en las Escuelas de Educación Media Superior (ENEIVEMS), que se llevó a cabo en una muestra representativa de estudiantes de educación media superior de las 32 entidades de República Mexicana, durante los meses de noviembre y diciembre de 2009. Es una encuesta transversal, probabilística, estratificada y por conglomerados. La estratificación de las unidades de muestreo se realizó, en cada estado, según las categorías de modalidad-plantel que agrupa a los planteles como: Bachillerato General, Bachillerato Tecnológico y Profesional Técnico.

\section{Orientación sexual}

Para evaluar la orientación sexual se formaron crearon dos variables: noviazgo con personas del mismo sexo y relaciones sexuales con personas del mismo sexo. La primera variable se formó a partir de las preguntas “¿Cuántos novios has tenido en toda tu vida?” y “ $i$ Tu novio(a) actual es de tu mismo sexo?". A partir de las respuestas se formaron tres grupos: a) no ha tenido noviazgo; 
b) noviazgo con persona de distinto sexo; y c) noviazgo con personas del mismo sexo. La segunda variable se formó a partir de las preguntas " $¿$ Has tenido alguna vez relaciones sexuales?” y “ $i$ Has tenido relaciones sexuales con personas de tu mismo sexo?" Se formaron tres grupos: a) no ha tenido relaciones sexuales; b) ha tenido relaciones sexuales con personas de distinto sexo; y c) ha tenido relaciones sexuales con personas del mismo sexo.

\section{Variables mediadoras}

Para evaluar las experiencias de violencia en la escuela se incluyeron frases que describían la relación del estudiante con sus compañeros de escuela de los últimos 12 meses. Se le indicaba al estudiante que pensara en qué grado cada una de ellas lo describían y seleccionara la que mejor lo definiera. Existían tres opciones de respuesta, las cuales fueron dicotomizadas en: a) "nunca"; y b) "a veces" y "a menudo". A partir de los resultados del análisis de componentes principales se crearon tres variables: a) violencia psicológica en la escuela (preguntas "me han ignorado" y "me han rechazado"); b) violencia verbal en la escuela (ítems "me han insultado" y "me han llamado con apodos que me ofenden o ridiculizan"); y c) violencia extrema en la escuela (incluía cinco preguntas como "me obligan con amenazas a conductas o situaciones de carácter sexual en las que no quiero participar" o "me amenazan con armas”). Para las dos primeras variables se formaron tres grupos: nunca, a veces y muchas veces. Para violencia extrema en la escuela se crearon dos grupos: no experimento ninguna de las cinco y experimentó algunas de las cinco. Los resultados de los análisis de componentes principales de todas las variables pueden ser solicitados a los autores.

Se incluyeron una serie de preguntas sobre la violencia que alguna vez en su vida hubiera recibido de algún familiar (padre, madre, hermanos o tíos). Las cuatro opciones de respuesta fueron dicotomizadas en: a) "nunca" y b) "pocas veces", "regularmente" y "muchas veces". Con análisis factorial se identificaron dos componentes: a) Violencia familiar verbal y física (incluía seis preguntas como " $i$ te han insultado?" o “ $i$ te han amenazado con golpearte?”) y b) violencia familiar extrema (abarcaba siete preguntas como " $i$ te han amenazado con alguna navaja o cuchillo o machete o con algún objeto similar?” o “ite han amenazado con matarte o amenazado con alguna pistola o rifle o con algún arma similar?"). Posteriormente, se sumaron las respuestas de cada factor quedando cuatro grupos para la primera variables: a) nunca; b) a veces; c) regularmente; y d) muchas veces. Para la segunda se definieron dos grupos: a) no ha experimentado ninguna de las siete y b) ha experimentado alguna de las siete formas de violencia.

Para evaluar las actitudes hacia la homosexualidad se le indicaba al entrevistado que pensara en qué grado cada una de ellas lo describían y rodeara con un círculo la puntuación en que mejor lo definiera, teniendo en cuenta que 0 refleja ningún acuerdo y el 7 refleja máximo acuerdo. Se analizaron tres preguntas: "Se debería reconocer el derecho de las parejas homosexuales a casarse legalmente"; "Despedir de su empleo a un maestro por ser homosexual es una violación a sus derechos” y “iHasta qué punto te gustaría tener como compañero en la escuela a un alumno(a) homosexual?". Para las tres variables las respuestas fueron recodificadas a ningún acuerdo (respuesta 0 y 1), de acuerdo (respuestas de 2 a 5), y máximo acuerdo (respuestas 6 y 7). Se consideró que estas preguntas podrían proporcionar información sobre la internalización del estigma en los adolescentes que no eran heterosexuales.

\section{Eventos de salud mental}

Los eventos estudiados fueron: síntomas de depresión, autoestima, ideación e intento de suicidio, consumo de tabaco, alcohol y drogas. La existencia de síntomas de depresión fue evaluada mediante la Escala de Depresión del Centro de Estudios Epidemiológicos, la cual en la población mexicana tiene propiedades psicométricas adecuadas 7. A partir de análisis factorial se crearon dos variables: a) síntomas de depresión: incluía trece afirmaciones como "sentí como si como si no pudiera quitarme de encima la tristeza, ni siquiera con la ayuda de mis familiares o amigos", "me sentí solo(a)" o "tenía ganas de llorar" y b) vitalidad, se generó a partir de cuatro afirmaciones como "fui feliz" o "disfruté de la vida". Existían cuatro opciones de respuesta (nunca, a veces, regularmente y muchas veces) a las cuales se les asignó una puntuación de cero a tres y las respuestas a las preguntas de cada factor fueron sumadas.

Para valorar el autoestima se utilizó la escala de Autoconcepto Forma $A$ 8. A partir de los resultados de análisis factorial se formaron cuatro variables: a) autoestima en el área escolar, conteniendo seis frases como "hago bien los trabajos escolares" o "mis profesores(as) me consideran un buen estudiante"; b) relaciones positivas en el área familiar, incluyendo cuatros frases como "mi familia me ayudaría en cualquier tipo de problema" o "mis padres me dan confianza"; c) baja auto estima, se incluyeron seis frases como "muchas cosas me ponen nervioso(a)" o "cuando los ma- 
yores me dicen algo me pongo muy nervioso(a)"; y d) relaciones positivas con amigos incluía seis frases como "mis amigos me estiman" o "tengo muchos amigos(as)". Existían cinco opciones de respuesta (nunca, pocas veces, algunas veces, muchas veces y siempre) a las cuales se les asignó una puntuación de cero a cuatro y posteriormente las respuestas de cada factor fueron sumadas.

La existencia de ideación suicida se evaluó con respuesta positiva a por lo menos una de las siguientes preguntas: ¿̇has tenido la sensación de que no vale la pena vivir?, ¿has vivido situaciones ante las que has deseado dejar de existir? y ¿has pensado que vale más morir que vivir?. Se consideró que los adolescentes habían intentado suicidarse, si respondieron positivamente a por lo menos una de las siguientes preguntas: ¿has estado a punto de intentar quitarte la vida? y

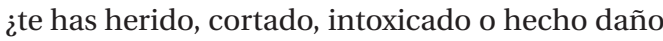
con el fin de quitarte la vida?. Estas preguntas de conducta suicida fueron tomadas de la Encuesta Nacional de Adicciones de México 9.

Considerando el trabajo de un grupo internacional sobre consumo de tabaco en adolescentes 10 , se formaron tres variables: a) consumo alguna vez en la vida de tabaco: adolescentes que por lo menos habían fumado por lo menos cien cigarrillos (5 cajetillas) de tabaco durante toda su vida?"; b) consumo actual de tabaco: fumado tabaco en los últimos en los últimos 30 días; y c) consumo elevado de tabaco: fumar 6 o más cigarrillos al día en los últimos 30 días.

Considerando la literatura 11,12, para valorar el consumo de alcohol se formaron las siguientes variables: a) consumo de alcohol alguna vez en la vida; b) consumo excesivo de alcohol: tomar cinco copas o más en una ocasión siete o más veces al año; c) embriaguez: sentirse embriagado (dificultad para mantenerte en pie o para caminar) seis o más veces al año; y d) consumo problemático de alcohol: haber tenido experiencias desagradables por el consumo de alcohol.

Para valorar el consumo de drogas los entrevistados tenían que responder cuáles drogas y con qué frecuencia habían consumido en su vida. Se formaron dos variables: a) consumo de drogas médicas: opiáceos, tranquilizantes, sedantes o barbitúricos, y anfetaminas o estimulantes y b) consumo de drogas ilegales: marihuana, cocaína en polvo, crack, alucinógenos, inhalables, heroína u opio y metanfetaminas. Las opciones de respuesta para las dos variables fueron dicotomizadas (nunca y pocas veces versus a veces, varias veces, con frecuencia y muchas veces). Se consideró que existía consumo cuando habían consumido por lo menos una droga.

También se evaluaron características sociodemográficas de los adolescentes como edad, sexo, grado que cursaban y ocupación (opciones: sólo estudia, principalmente estudia y hace algún trabajo, principalmente trabaja y además estudia, estudia y está buscando trabajo los encuestados). La escolaridad del padre y de la madre fueron divididas en: educación primaria y menos, secundaria, preparatoria, licenciatura y más.

\section{Análisis estadístico}

Para el análisis estadístico se utilizó el programa Stata versión 11 (Stata Corp., College Station, Estados Unidos). Las estimaciones se hicieron utilizando los comandos de encuestas (svy), con lo cual se consideró el diseño complejo de la ENEIVEMS (i.e. probabilística, estratificada y por conglomerados). Se obtuvieron las frecuencias absolutas y relativas de las variables bajo estudio (Tablas 1 y 2). Cuando se hicieron comparaciones entre grupos se utilizó estadística chicuadrada para variables categóricas y la prueba de $t$ de student para muestras independientes. Posteriormente, se estimaron modelos de regresión logística o lineales, en los cuales las variables dependientes fueron los eventos de salud; mientras que las variables independientes fueron los indicadores de orientación sexual (Tabla 3). Estos modelos fueron ajustados por edad y sexo del adolescente y escolaridad de la madre.

Se estimaron modelos de regresión logística (para el caso de la ideación y el intento de suicidio, el consumo problemático de alcohol y el consumo de drogas médicas) y lineal (para los síntomas de depresión) con la finalidad de evaluar si las experiencias de violencia y la actitud hacia la homosexualidad mediaban la asociación entre la orientación sexual y los indicadores de salud mental. Un mediador debe cumplir con ciertas premisas 13,14: a) ser más frecuente en el grupo en desventaja; b) estar asociado con los eventos de salud estudiados; y c) al ser incorporado en los modelos de regresión debe resultar en disminución de las estimaciones de las asociaciones entre la exposición (i.e. orientación sexual) y el evento (indicadores de salud mental). Para evaluar la primera premisa se estimó la distribución de la violencia en la escuela y la familia y la actitud hacia la homosexualidad, de acuerdo con la orientación sexual (Tabla 4). Para evaluar la segunda premisa se estimaron las diferencias en los eventos de salud de acuerdo con la violencia en la escuela y la familia y la actitud hacia la homosexualidad. En estos últimos casos sólo se consideraron los eventos en salud en los que habían existido diferencias referidas a la orientación sexual (Tabla 3 ).

Para la tercera premisa se estimaron modelos de regresión utilizando el comando khb pa- 
Características sociodemográficas de acuerdo con la orientación sexual.

\begin{tabular}{|c|c|c|c|c|c|c|c|c|}
\hline & \multicolumn{4}{|c|}{ Noviazgo } & \multicolumn{4}{|c|}{ Relaciones sexuales } \\
\hline & $\begin{array}{c}\text { No ha tenido } \\
\text { noviazgo } \\
{[n=1.006 \text { * }(\%)}\end{array}$ & $\begin{array}{l}\text { Con personas de } \\
\text { sexo distinto } \\
{[n=7.444 \text { *] (\%) }}\end{array}$ & $\begin{array}{l}\text { Con personas } \\
\text { del mismo sexo } \\
{\left[n=319{ }^{\star}\right](\%)}\end{array}$ & $\begin{array}{l}\text { Valor } \\
\text { del p }\end{array}$ & $\begin{array}{c}\text { No ha tenido } \\
\text { relaciones } \\
\text { sexuales } \\
{\left[n=6,437^{*}\right](\%)}\end{array}$ & $\begin{array}{l}\text { Con personas de } \\
\text { sexo distinto } \\
{[\mathrm{n}=2.227 \text { * }(\%)}\end{array}$ & $\begin{array}{l}\text { Con personas } \\
\text { del mismo sexo } \\
{\left[n=122{ }^{\star}\right](\%)}\end{array}$ & $\begin{array}{l}\text { Valor } \\
\text { del p }\end{array}$ \\
\hline \multicolumn{9}{|l|}{ Edad (años) } \\
\hline $14-16$ & 67,0 & 56,5 & 51,3 & 0,000 & 64,1 & 41,0 & 50,8 & 0,000 \\
\hline $17-19$ & 33,0 & 43,5 & 48,7 & & 35,9 & 59,0 & 49,2 & \\
\hline \multicolumn{9}{|l|}{ Sexo } \\
\hline Hombre & 45,6 & 47,1 & 41,7 & 0,509 & 40,6 & 61,5 & 71,2 & 0,000 \\
\hline Mujer & 54,4 & 52,9 & 58,3 & & 59,4 & 38,5 & 28,8 & \\
\hline \multicolumn{9}{|l|}{ Grado que cursan } \\
\hline Primer año & 50,5 & 38,2 & 33,2 & 0,000 & 43,3 & 29,5 & 31,5 & 0,000 \\
\hline Segundo año & 25,4 & 32,4 & 30,0 & & 30,6 & 34,2 & 35,4 & \\
\hline Tercer año & 24,1 & 29,4 & 36,8 & & 26,1 & 36,3 & 33,1 & \\
\hline \multicolumn{9}{|l|}{ Ocupación } \\
\hline Sólo estudia & 74,7 & 67,4 & 67,2 & 0,001 & 73,0 & 56,3 & 57,9 & 0,000 \\
\hline $\begin{array}{l}\text { Principalmente } \\
\text { estudia y hace } \\
\text { algún trabajo }\end{array}$ & 15,8 & 18,0 & 13,4 & & 15,8 & 22,3 & 19,0 & \\
\hline $\begin{array}{l}\text { Principalmente } \\
\text { trabaja y además } \\
\text { estudia }\end{array}$ & 2,5 & 3,4 & 3,2 & & 2,6 & 4,8 & 4,3 & \\
\hline $\begin{array}{l}\text { Estudia y está } \\
\text { buscando trabajo } \\
\text { Escolaridad del } \\
\text { padre }\end{array}$ & 7,0 & 11,2 & 16,2 & & 8,6 & 16,6 & 18,8 & \\
\hline Primaria y menos & 39,9 & 29,4 & 25,0 & 0,000 & 33,1 & 23,7 & 22,2 & 0,001 \\
\hline Secundaria & 22,9 & 26,9 & 31,7 & & 26,5 & 26,5 & 26,7 & \\
\hline Preparatoria & 24,1 & 27,6 & 21,4 & & 25,5 & 31,2 & 25,7 & \\
\hline $\begin{array}{l}\text { Licenciatura y } \\
\text { más }\end{array}$ & 13,1 & 16,1 & 21,9 & & 14,9 & 18,6 & 25,4 & \\
\hline \multicolumn{9}{|l|}{$\begin{array}{l}\text { Escolaridad de la } \\
\text { madre }\end{array}$} \\
\hline Primaria y menos & 41,7 & 30,6 & 27,0 & 0,000 & 34,1 & 25,8 & 26,9 & 0,000 \\
\hline Secundaria & 25,6 & 32,2 & 31,7 & & 31,5 & 31,4 & 26,4 & \\
\hline Preparatoria & 21,3 & 24,7 & 24,2 & & 22,5 & 28,5 & 35,4 & \\
\hline $\begin{array}{l}\text { Licenciatura y } \\
\text { más }\end{array}$ & 11,4 & 12,4 & 17,1 & & 11,9 & 14,3 & 11,3 & \\
\hline
\end{tabular}

Nota: proporciones (\%) ponderadas de acuerdo con el diseño complejo de la Encuesta Nacional Sobre Exclusión, Intolerancia y Violencia en las Escuelas de Educación Media Superior.

* Sin ponderar.

ra Stata desarrollado por Karlson, Holm y Breen (Tabla 5) 13,14. Este procedimiento tiene la ventaja que permite modelar variables continuas (con la función regress) o categóricas (función logit); además, es posible considerar el diseño muestral, pues al hacer estimaciones se pueden incorporar los ponderadores y ajustar los errores estándares por la existencia de los conglomerados. Además, proporciona estimaciones sin sesgo de las contribuciones de variables mediadoras, tanto categóricas como continuas. Otra ventaja es que permite incluir variables confusoras de efecto. Sólo se modelaron los eventos en los que existieron diferencias referentes a la orientación sexual 


\begin{tabular}{|c|c|c|c|c|c|c|c|c|}
\hline & \multicolumn{4}{|c|}{ Noviazgo } & \multicolumn{4}{|c|}{ Relaciones sexuales } \\
\hline & $\begin{array}{c}\text { No ha tenido } \\
\text { noviazgo }\end{array}$ & $\begin{array}{l}\text { Con personas } \\
\text { de distinto } \\
\text { sexo }\end{array}$ & $\begin{array}{l}\text { Con personas } \\
\text { del mismo } \\
\text { sexo }\end{array}$ & & NRS & $\begin{array}{l}\text { Con personas } \\
\text { de distinto } \\
\text { sexo }\end{array}$ & $\begin{array}{l}\text { Con personas } \\
\text { del mismo } \\
\text { sexo }\end{array}$ & \\
\hline & M & M & M & & M & M & M & \\
\hline Síntomas de depresión & $9,8 a, b$ & 10,7 a & $12,0 \mathrm{~b}$ & & $10,5 b$ & $11,0 \mathrm{c}$ & $13,4 b, c$ & \\
\hline Vitalidad & 6,5 & 6,5 & 6,4 & & 6,5 & 6,5 & 6,8 & \\
\hline Autoestima en el área escolar & 14,6 a & 13,4 a & 14,1 & & 13,9 a & 12,8 a & 13,5 & \\
\hline Relaciones positivas en el área familiar & 15,4 a & 14,5 a & 14,5 & & 14,9 a & 14,0 & 13,3 a & \\
\hline Baja autoestima & 9,1 a & 8,7 a & 9,2 & & 8,9 a & 8,4 a & 8,7 & \\
\hline \multirow[t]{2}{*}{ Relaciones positivas con amigos } & $16,1 \mathrm{a}, \mathrm{b}$ & $17,3 a, c$ & $17,9 b, c$ & & 17,1 & 17,4 & 17,4 & \\
\hline & $\%$ & $\%$ & $\%$ & Valor del $p$ & $\%$ & $\%$ & $\%$ & $\begin{array}{l}\text { Valor } \\
\text { del p }\end{array}$ \\
\hline Ideación suicida & 36,9 & 43,4 & 49,5 & 0,008 & 41,3 & 45,9 & 62,0 & 0,000 \\
\hline Intento de suicidio & 5,8 & 12,2 & 20,5 & 0,000 & 9,9 & 15,6 & 31,5 & 0,000 \\
\hline $\begin{array}{l}\text { Consumo de tabaco: alguna vez en } \\
\text { la vida }\end{array}$ & 40,9 & 66,2 & 73,7 & 0,000 & 56,6 & 80,9 & 83,5 & 0,000 \\
\hline Consumo actual de tabaco & 3,3 & 14,4 & 15,2 & 0,000 & 7,4 & 27,1 & 31,5 & 0,000 \\
\hline Consumo elevado de tabaco & 1,3 & 5,9 & 4,8 & 0,000 & 2,5 & 12,3 & 11,5 & 0,000 \\
\hline $\begin{array}{l}\text { Consumo de alcohol: alguna vez en } \\
\text { la vida }\end{array}$ & 32,5 & 64,4 & 72,0 & 0,000 & 54,2 & 78,1 & 81,0 & 0,000 \\
\hline Consumo excesivo de alcohol & 12,1 & 38,6 & 45,3 & 0,000 & 28,0 & 55,6 & 52,1 & 0,000 \\
\hline Consumo de alcohol: embriaguez & 2,6 & 14,0 & 19,4 & 0,000 & 8,1 & 24,5 & 19,9 & 0,000 \\
\hline Consumo problemático de alcohol & 4,8 & 17,9 & 24,3 & 0,000 & 12,1 & 27,8 & 34,3 & 0,000 \\
\hline Consumo de drogas médicas & 3,4 & 6,8 & 11,9 & 0,000 & 5,2 & 9,9 & 12,0 & 0,000 \\
\hline Consumo de drogas ilegales & 2,1 & 6,8 & 4,1 & 0,000 & 3,4 & 13,2 & 12,4 & 0,000 \\
\hline
\end{tabular}

Superíndices $(a, b$ y $c)$ similares indican diferencias estadísticamente significativas $(p<0,050)$.

Nota: medias (M) y prevalencias (\%) ponderadas, de acuerdo con el diseño complejo de la Encuesta Nacional Sobre Exclusión, Intolerancia y Violencia en las Escuelas de Educación Media Superior.

(Tabla 3). En los modelos reducidos se evaluó el efecto de haber tenido un noviazgo o relaciones sexuales con personas del mismo sexo sobre la probabilidad de presentar los eventos de salud. El grupo de referencia fueron los adolescentes que habían tenido un noviazgo o relaciones sexuales con personas de distinto sexo. En los modelos completos se incluyeron las variables mediadoras en las que existieron diferencias estadísticamente significativas de acuerdo con la orientación sexual. En todos los modelos se incluyó como covariables la edad y el sexo de los adolescentes, así como la escolaridad de la madre.

El proyecto fue aprobado por la Comisión de Investigación del Instituto Nacional de Salud Pública de México. Se obtuvo el consentimiento informado en los estudiantes mayores de 18 años y el asentimiento en el caso de los menores de edad con el consentimiento de sus padres.

\section{Resultados}

El 3,4\% ( $n=319)$ de los adolescentes reportaron haber tenido un noviazgo con una persona de su mismo sexo, el 85,9\% ( $\mathrm{n}=7.444)$ tuvo noviazgo con una persona de distinto sexo y $10,7 \%$ no había tenido novio $(\mathrm{n}=1.006)$. Respecto a las relaciones sexuales, las proporciones fueron: $1,3 \%$ $(n=122), 28,0 \%(n=2.227)$ y $70,7 \%(n=6.437)$, respectivamente.

En la Tabla 1 se presentan las características sociodemográficas de acuerdo a la orientación sexual. De los adolescentes encuestados la mayoría contaba con 14 a 16 años de edad. En los adolescentes que habían tenido un noviazgo con una persona de su mismo sexo existieron proporciones más altas de varones, de adolescentes que estudiaban y estaban buscando trabajo, y cuyos padres contaban con escolaridad de licenciatura o más. 
Modelos de regresión lineal y logística teniendo de acuerdo, como variables dependientes, los eventos de salud y como variable independiente la orientación sexual.

\begin{tabular}{|c|c|c|c|c|c|c|c|c|}
\hline & \multicolumn{4}{|c|}{ Noviazgo * } & \multicolumn{4}{|c|}{ Relaciones sexuales ** } \\
\hline & \multicolumn{2}{|c|}{ No ha tenido noviazgo } & \multicolumn{2}{|c|}{$\begin{array}{c}\text { Con personas del mismo } \\
\text { sexo }\end{array}$} & \multicolumn{2}{|c|}{$\begin{array}{c}\text { No ha tenido relaciones } \\
\text { sexuales }\end{array}$} & \multicolumn{2}{|c|}{$\begin{array}{c}\text { Con personas del mismo } \\
\text { sexo }\end{array}$} \\
\hline & \multicolumn{8}{|c|}{ Modelos de regresión lineal } \\
\hline & B & IC95\% & B & IC95\% & B & IC95\% & B & IC95\% \\
\hline Síntomas de depresión & $-0,87$ & $-1,68 ;-0,06$ & 1,09 & $-0,45 ; 2,64$ & $-1,28$ & $-1,85 ;-0,71$ & 2,81 & 1,$46 ; 4,16$ \\
\hline Vitalidad & 0,02 & $-0,30 ; 0,34$ & $-0,16$ & $-0,63 ; 0,31$ & 0,03 & $-0,13 ; 0,19$ & 0,29 & $-0,52 ; 1,11$ \\
\hline $\begin{array}{l}\text { Autoestima en el área } \\
\text { escolar }\end{array}$ & 1,22 & 0,$73 ; 1,72$ & 0,53 & $-0,39 ; 1,47$ & 1,07 & 0,$66 ; 1,48$ & 0,72 & $-1,35 ; 2,80$ \\
\hline $\begin{array}{l}\text { Relaciones positivas en el } \\
\text { área familiar }\end{array}$ & 0,86 & 0,$20 ; 1,52$ & $-0,02$ & $-0,76 ; 0,70$ & 1,02 & 0,$64 ; 1,40$ & $-0,69$ & $-2,65 ; 1,26$ \\
\hline Baja autoestima & 0,35 & $-0,02 ; 0,73$ & 0,36 & $-0,62 ; 1,35$ & 0,10 & $-0,26 ; 0,47$ & 0,56 & $-0,62 ; 1,74$ \\
\hline \multirow{3}{*}{$\begin{array}{l}\text { Relaciones positivas con } \\
\text { amigos }\end{array}$} & $-1,17$ & $-1,70 ;-0,64$ & 0,44 & $-0,06 ; 0,94$ & $-0,34$ & $-0,82 ; 0,13$ & 0,04 & $-1,84 ; 1,93$ \\
\hline & \multicolumn{8}{|c|}{ Modelos de regresión logística } \\
\hline & RM & IC95\% & RM & IC95\% & RM & IC95\% & RM & IC95\% \\
\hline Ideación suicida & 0,74 & 0,$59 ; 0,93$ & 1,23 & 0,$89 ; 1,70$ & 0,67 & 0,$59 ; 0,75$ & 2,09 & 1,$24 ; 3,50$ \\
\hline Intento de suicidio & 0,43 & 0,$29 ; 0,65$ & 1,78 & 1,$21 ; 2,62$ & 0,46 & 0,$36 ; 0,58$ & 2,86 & 1,$75 ; 4,68$ \\
\hline $\begin{array}{l}\text { Consumo de tabaco: } \\
\text { alguna vez en la vida }\end{array}$ & 0,36 & 0,$27 ; 0,48$ & 1,46 & 0,$99 ; 2,13$ & 0,33 & 0,$28 ; 0,38$ & 1,18 & 0,$61 ; 2,29$ \\
\hline $\begin{array}{l}\text { Consumo actual de } \\
\text { tabaco }\end{array}$ & 0,22 & 0,$13 ; 0,38$ & 1,08 & 0,$66 ; 1,74$ & 0,26 & 0,$21 ; 0,31$ & 1,22 & 0,$70 ; 2,15$ \\
\hline $\begin{array}{l}\text { Consumo elevado de } \\
\text { tabaco }\end{array}$ & 0,24 & 0,$10 ; 0,56$ & 0,81 & 0,$42 ; 1,57$ & 0,23 & 0,$17 ; 0,30$ & 0,91 & 0,$44 ; 1,84$ \\
\hline $\begin{array}{l}\text { Consumo de alcohol: } \\
\text { alguna vez en la vida }\end{array}$ & 0,28 & 0,$22 ; 0,37$ & 1,36 & 0,$94 ; 1,97$ & 0,35 & 0,$29 ; 0,42$ & 1,27 & 0,$72 ; 2,23$ \\
\hline $\begin{array}{l}\text { Consumo excesivo de } \\
\text { alcohol }\end{array}$ & 0,23 & 0,$17 ; 0,33$ & 1,28 & 0,$96 ; 1,71$ & 0,35 & 0,$28 ; 0,43$ & 0,89 & 0,$46 ; 1,71$ \\
\hline $\begin{array}{l}\text { Consumo de alcohol: } \\
\text { embriaguez }\end{array}$ & 0,17 & 0,$09 ; 0,32$ & 1,47 & 0,$85 ; 2,55$ & 0,29 & 0,$23 ; 0,38$ & 0,77 & 0,$44 ; 1,33$ \\
\hline $\begin{array}{l}\text { Consumo problemático } \\
\text { de alcohol }\end{array}$ & 0,25 & 0,$16 ; 0,37$ & 1,46 & 1,$05 ; 2,02$ & 0,39 & 0,$33 ; 0,46$ & 1,39 & 0,$70 ; 2,74$ \\
\hline $\begin{array}{l}\text { Consumo de drogas } \\
\text { médicas }\end{array}$ & 0,48 & 0,$31 ; 0,74$ & 1,85 & 1,$10 ; 3,10$ & 0,50 & 0,$39 ; 0,64$ & 1,23 & 0,$55 ; 2,74$ \\
\hline $\begin{array}{l}\text { Consumo de drogas } \\
\text { ilegales }\end{array}$ & 0,31 & 0,$19 ; 0,50$ & 0,60 & 0,$31 ; 1,13$ & 0,27 & 0,$21 ; 0,35$ & 0,89 & 0,$45 ; 1,75$ \\
\hline
\end{tabular}

B: coeficiente de regresión; IC95\%: intervalo de confianza al 95\%; OR: razón de momios.

Modelos ajustados por edad, sexo y escolaridad de la madre. Los modelos fueron ponderados de acuerdo con el diseño complejo de la encuesta.

* El grupo de referencia fueron los adolescentes que habían tenido un noviazgo con personas de distinto sexo;

** El grupo de referencia fueron los adolescentes que habían tenido relaciones sexuales con personas de distinto sexo.

En la Tabla 2 se muestran las diferencias en eventos de salud de acuerdo a orientación sexual. En vitalidad no existieron diferencias de acuerdo con haber tenido o no noviazgo o el haber tenido relaciones sexuales o no. Los adolescentes con noviazgo con una persona de su mismo sexo presentaron más síntomas de depresión y pun- tuaciones más altas en las relaciones positivas con los amigos. En comparación con los adolescentes que no habían tenido noviazgo (o no habían tenido relaciones sexuales), aquellos que sí lo habían tenido (o habían tenido relaciones sexuales) ya sea con personas de su mismo sexo o con personas de distinto sexo presentaron 
Diferencias en violencia y actitudes hacia la homosexualidad de acuerdo con la orientación sexual.

\begin{tabular}{|c|c|c|c|c|c|c|c|c|c|}
\hline & \multirow[t]{2}{*}{ Total (\%) } & \multicolumn{4}{|c|}{ Noviazgo } & \multicolumn{4}{|c|}{ Relaciones sexuales } \\
\hline & & $\begin{array}{c}\text { No ha } \\
\text { tenido } \\
\text { noviazgo } \\
\text { (\%) }\end{array}$ & $\begin{array}{c}\text { Con } \\
\text { personas } \\
\text { de distinto } \\
\text { sexo (\%) }\end{array}$ & $\begin{array}{c}\text { Con } \\
\text { personas } \\
\text { del mismo } \\
\text { sexo (\%) }\end{array}$ & $\begin{array}{l}\text { Valor } \\
\text { del } p\end{array}$ & $\begin{array}{c}\text { No ha } \\
\text { tenido } \\
\text { relaciones } \\
\text { sexuales (\%) }\end{array}$ & $\begin{array}{c}\text { Con } \\
\text { personas } \\
\text { de distinto } \\
\text { sexo (\%) }\end{array}$ & $\begin{array}{c}\text { Con } \\
\text { personas } \\
\text { del mismo } \\
\text { sexo }(\%)\end{array}$ & $\begin{array}{l}\text { Valor } \\
\text { del } p\end{array}$ \\
\hline \multicolumn{10}{|c|}{ Violencia familiar extrema } \\
\hline Ausente & 93,1 & 94,8 & 93,0 & 90,6 & 0,088 & 94,0 & 91,0 & 81,7 & 0,000 \\
\hline Presente & 6,9 & 5,2 & 7,0 & 9,4 & & 6,0 & 9,0 & 18,3 & \\
\hline \multicolumn{10}{|c|}{ Violencia familiar verbal y física } \\
\hline Nunca & 29,5 & 33,8 & 29,0 & 28,2 & 0,093 & 31,2 & 25,5 & 22,9 & 0,000 \\
\hline A veces & 33,1 & 34,6 & 33,2 & 27,9 & & 34,5 & 30,3 & 17,5 & \\
\hline Regularmente & 20,7 & 17,8 & 20,8 & 25,8 & & 19,2 & 23,6 & 34,8 & \\
\hline Muchas veces & 16,7 & 13,7 & 17,0 & 18,1 & & 15,1 & 20,6 & 24,8 & \\
\hline \multicolumn{10}{|c|}{ Violencia psicológica en escuela } \\
\hline Nunca & 57,7 & 55,4 & 58,1 & 53,9 & 0,394 & 58,0 & 57,3 & 48,3 & 0,023 \\
\hline A veces & 25,2 & 24,6 & 25,2 & 26,6 & & 25,5 & 24,0 & 34,5 & \\
\hline Muchas veces & 17,1 & 20,0 & 16,7 & 19,5 & & 16,5 & 18,7 & 17,2 & \\
\hline \multicolumn{10}{|c|}{ Violencia verbal en escuela } \\
\hline Nunca & 47,8 & 51,1 & 47,8 & 39,3 & 0,083 & 49,7 & 43,8 & 33,7 & 0,000 \\
\hline A veces & 28,6 & 25,9 & 28,8 & 32,3 & & 28,1 & 30,0 & 26,3 & \\
\hline Muchas veces & 23,6 & 23,0 & 23,4 & 28,4 & & 22,2 & 26,2 & 40,0 & \\
\hline \multicolumn{10}{|c|}{ Violencia extrema en escuela } \\
\hline Ausente & 91,3 & 92,7 & 91,3 & 85,5 & 0,051 & 91,9 & 90,6 & 69,9 & 0,000 \\
\hline Presente & 8,7 & 7,3 & 8,7 & 14,5 & & 8,1 & 9,4 & 30,1 & \\
\hline \multicolumn{10}{|c|}{$\begin{array}{l}\text { Opinión sobre: se debería reconocer el } \\
\text { derecho de las parejas homosexuales a } \\
\text { casarse legalmente }\end{array}$} \\
\hline Ningún acuerdo & 46,4 & 56,5 & 45,5 & 37,2 & 0,000 & 47,2 & 45,2 & 32,7 & 0,001 \\
\hline De acuerdo & 27,7 & 21,6 & 28,6 & 23,5 & & 27,4 & 28,9 & 15,9 & \\
\hline Máximo acuerdo & 25,9 & 21,9 & 25,9 & 39,3 & & 25,4 & 25,9 & 51,4 & \\
\hline $\begin{array}{l}\text { Opinión sobre: desp } \\
\text { a un maestro por ser } \\
\text { una violación a sus o }\end{array}$ & & & & & & & & & \\
\hline Ningún acuerdo & 54,7 & 54,3 & 54,6 & 58,0 & 0,667 & 54,5 & 55,3 & 51,9 & 0,687 \\
\hline De acuerdo & 13,9 & 13,3 & 14,2 & 10,1 & & 14,0 & 14,0 & 9,5 & \\
\hline Máximo acuerdo & 31,4 & 32,4 & 31,2 & 31,9 & & 31,5 & 30,7 & 38,6 & \\
\hline \multicolumn{10}{|c|}{$\begin{array}{l}\text { Opinión sobre: ¿hasta qué punto te } \\
\text { gustaría tener como compañero en la } \\
\text { escuela a un alumno(a) homosexual? }\end{array}$} \\
\hline Ningún acuerdo & 30,3 & 36,8 & 30,0 & 16,3 & 0,000 & 30,8 & 29,5 & 21,8 & 0,001 \\
\hline De acuerdo & 36,8 & 32,1 & 37,6 & 32,7 & & 36,4 & 38,7 & 19,5 & \\
\hline Máximo acuerdo & 32,9 & 31,1 & 32,4 & 51,0 & & 32,8 & 31,8 & 58,7 & \\
\hline
\end{tabular}

Nota: proporciones (\%) ponderadas de acuerdo con el diseño complejo de la Encuesta Nacional Sobre Exclusión, Intolerancia y Violencia en las Escuelas de Educación Media Superior.

mayor prevalencia de ideación suicida, intento de suicidio, consumo de tabaco alguna vez en la vida, consumo actual de tabaco, consumo de alcohol alguna vez en la vida, consumo excesivo de alcohol, embriaguez, consumo problemático de alcohol y consumo de drogas.

Respecto a los adolescentes que habían tenido un noviazgo o relaciones sexuales con perso- 
Modelos de regresión para evaluar la contribución de variables mediadoras. La variable independiente es la orientación sexual y las variables dependientes los eventos de salud.

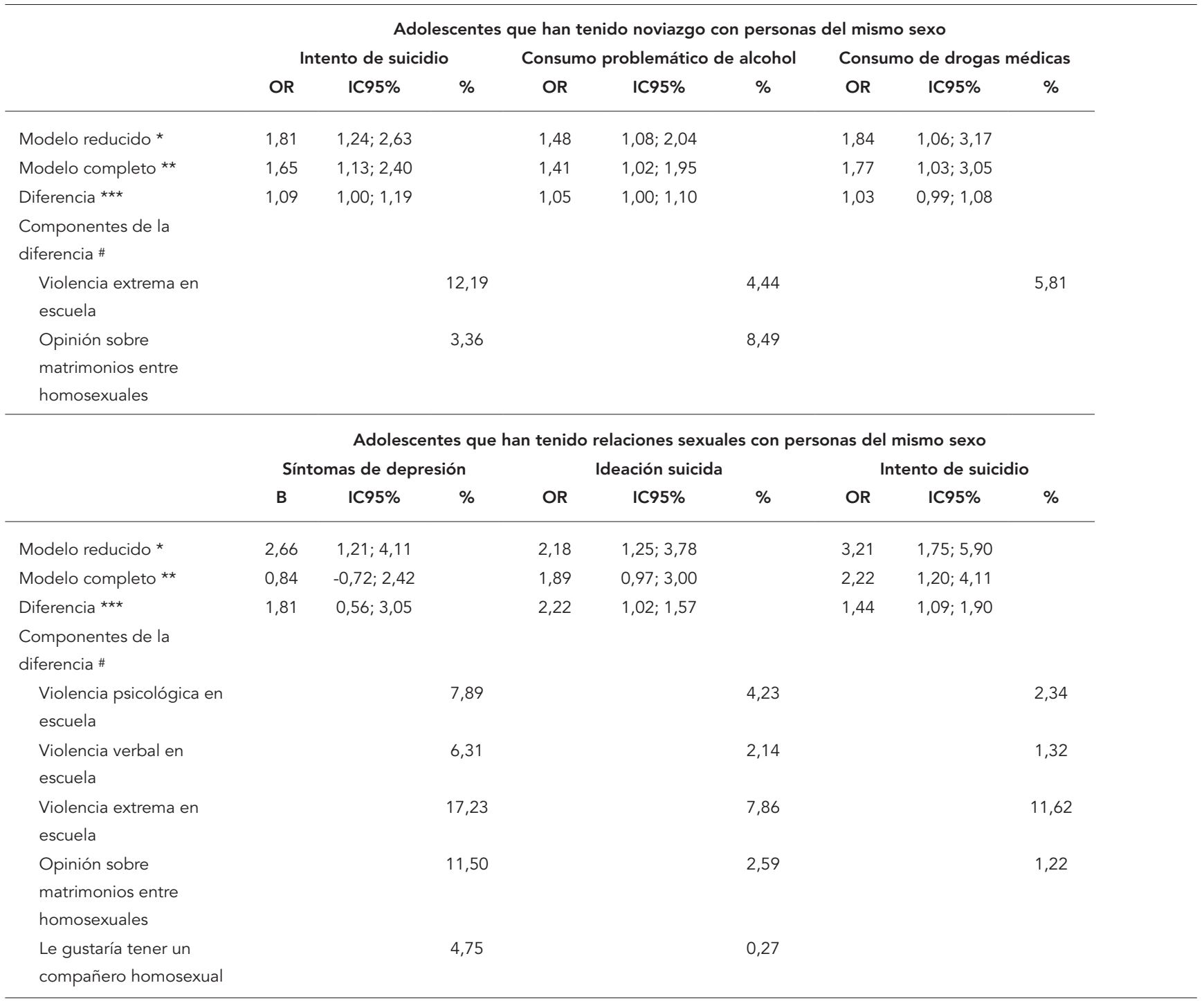

B: coeficiente de regresión en un modelo de regresión lineal; IC95\%: intervalo de confianza al 95\%; OR: razón de momios en un modelo de regresión logística.

* Riesgo de los adolescentes que han tenido noviazgo o relaciones sexuales con personas del mismo sexo, respecto a los adolescentes que han tenido noviazgo o relaciones sexuales con personas de distinto sexo sin ajuste;

** Es el mismo riesgo del modelo reducido, pero ajustado por las variables que se postulan funcionan como mediadoras, las cuales corresponden a aquellas con valores en componentes de la diferencia. Tanto en el modelo reducido, como en el completo, se ajustó por edad, sexo y escolaridad de la madre;

*** Diferencias entre el modelo completo y reducido;

\# Es la contribución porcentual de cada variable a la diferencia entre modelos.

nas de distinto sexo, aquellos que no los habían tenido tuvieron mayor autoestima en el área escolar y relaciones positivas en la familia, y por el contrario; tuvieron menor probabilidad de tener ideación o intento suicida, consumo de tabaco, consumo de alcohol y consumo de drogas (Tabla 3). Al considerar como grupo de referencia a los adolescentes que habían tenido relaciones sexuales con personas de distinto sexo, aquellos que las habían tenido con personas de su mismo sexo tuvieron más síntomas de depresión y tuvieron mayor riesgo de ideación e intento de suicidio. Los adolescentes que habían tenido un noviazgo con persona de su mismo sexo tuvie- 
ron mayor probabilidad de experimentar intento de suicidio, consumo problemático de alcohol y consumir drogas médicas.

Las diferencias en violencia y actitudes hacia la homosexualidad según la orientación sexual se presentan en la Tabla 4. No existieron diferencias estadísticamente significativas entre los grupos formados según el tipo de noviazgo, respecto a la violencia familiar extrema, violencia familiar verbal y física, violencia psicológica en la escuela, violencia verbal en la escuela, violencia extrema en la escuela. La probabilidad de sufrir violencia en la escuela y la familia fue mayor en los adolescentes que habían tenido relaciones sexuales con personas de su mismo sexo. En los adolescentes que habían tenido algún noviazgo o relaciones sexuales con personas de su mismo sexo fue mayor la probabilidad de estar de acuerdo con el matrimonio entre personas del mismo sexo y en tener un compañero homosexual en la escuela.

Los adolescentes que muchas veces han experimentado violencia familiar y violencia en la escuela presentan más síntomas de depresión, respecto aquellos que no la han experimentado o la habían experimentado en menor grado (medias: 14,9 versus 7,5, $\mathrm{p}<0,050$ ) (datos no mostrados en tablas). En los adolescentes que habían sido víctimas de violencia verbal o física en la familia fueron más altas las prevalencias de ideación suicida $(61,4 \%$ versus $28,5 \%$ en los que no la habían sufrido, $\mathrm{p}<0,050$ ), intento de suicidio $(24,7 \%$ versus $5,9 \%, \mathrm{p}<0,050)$, consumo problemático de alcohol (25,1\% versus $10,6 \%$, p $<0,050)$ y consumo de drogas médicas $(10,8 \%$ versus $3,6 \%, \mathrm{p}<0,050$ ). La misma tendencia se observó con la violencia extrema en la familia y las dos formas de violencia en la escuela.

Los adolescentes que estaban de acuerdo con el matrimonio entre personas del mismo sexo presentaron más síntomas de depresión (medias: 12,3 versus $9,5, \mathrm{p}<0,050$ ) y prevalencias más elevadas de ideación suicida $(48,1 \%$ versus $39,5 \%, \mathrm{p}<0,050)$, intento de suicidio $(15,2 \%$ versus $10,7 \%, \mathrm{p}<0,050$ ) y consumo problemático de alcohol (19,2\% versus $14,7 \%, \mathrm{p}<0,050)$ (datos no mostrados en tablas). Las mismas tendencias se observaron en los adolescentes que reportaron no haber tenido relaciones sexuales. En los adolescentes que habían tenido relaciones sexuales con personas de distinto sexo no existieron diferencias. En el caso del grupo de adolescentes con noviazgo con personas de su mismo sexo, respecto a los adolescentes que estaban de acuerdo, aquellos que estaban no estaban de acuerdo y los que estaban en máximo acuerdo con el matrimonio entre personas del mismo sexo tuvieron tasas más altas de ideación (25,8\%, 71,8\% y $67,3 \%$, respectivamente, $\mathrm{p}<0,050$ ) e intento de suicidio $(8,1 \%, 42,9 \%$ y $31,7 \%$, respectivamente, $\mathrm{p}<0,050)$.

En la Tabla 5 se presentan los resultados de los análisis de mediación. Las experiencias de violencia y las actitudes a la homosexualidad prácticamente no explicaron las diferencias en intento de suicidio, consumo problemático de alcohol y de drogas médicas, según el tipo de noviazgo. La violencia verbal y física en la familia y la violencia extrema en la escuela fueron las variables que más explicaron las diferencias entre los adolescentes que habían tenido relaciones sexuales con persona de su mismo sexo y aquellos que habían tenido relaciones sexuales con persona de distinto sexo.

\section{Discusión}

Los adolescentes mexicanos matriculados en instituciones de educación media superior que han tenido noviazgos o relaciones sexuales con personas de su mismo sexo tuvieron un riesgo incrementando de experimentar síntomas depresivos, ideación e intento de suicidio y consumo problemático de alcohol. Los resultados de los modelos de regresión son coherentes con la hipótesis de que las diferencias en la morbilidad mental, asociadas con la orientación sexual (cuando ésta es evaluada con el indicador de actividad sexual), pueden ser explicadas por las experiencias de violencia en la familia y la escuela.

En un meta-análisis 4 de estudios realizados en Estados Unidos, Europa y Australasia se estimó que la población LBH tenía 2,04 veces mayor riesgo de presentar ideación suicida, 2,47 para intento de suicidio, 2,03 para depresión, 2,22 para dependencia al alcohol y 2,73 para dependencia a drogas. Estas estimaciones son similares a las obtenidas en los adolescentes de México: 2,09 para ideación suicida, 1,78 ó 2,86 para intento de suicidio, 1,46 para consumo problemático de alcohol y 1,85 para consumo de drogas médicas.

La persistencia de inequidades en salud relacionadas con la orientación sexual pueden ser producto del clima cultural, caracterizado por la intolerancia a las formas no dominantes de sexualidad y expresión de género. En México en los últimos años el porcentajes de personas que no quieren tener como vecinos a homosexuales han disminuido sustancialmente: de $60,0 \%$ en 1990 , a $44,6 \%$ en 2000 a $22,4 \%$ en 2004 (World Values Survey. http://www.worldvaluessurvey.org, accedido el 09/Oct/2012). Sin embargo, en la más reciente Encuesta Nacional de Discriminación del año 201015 se estimó que el 67\% de los mexi- 
canos no estuvo de acuerdo con el matrimonio entre personas del mismo sexo, el $68 \%$ consideró que a las mujeres lesbianas no se les debía permitir tener hijos y el $73 \%$ que a los hombres homosexuales no se les debía autorizar tenerlos. Estos datos muestran que cada vez serán menos frecuentes las formas abiertas o evidentes de homofobia; pero que la población mexicana sigue manteniendo una actitud negativa hacia lo que se considera una transgresión de la forma dominante de ejercer la sexualidad.

El clima cultural de intolerancia hacia las manifestaciones no dominantes de la sexualidad puede permear las relaciones interpersonales. De este modo, la población LBH sistemáticamente se enfrenta con más frecuencia a diferentes formas de violencia interpersonal 16 . En los adolescentes mexicanos, aquellos que tuvieron relaciones sexuales con personas de su mismo sexo tuvieron mayor probabilidad de haber sufrido violencia en la escuela y en la familia; aunque se observó la misma situación en aquellos que habían tenido un noviazgo con una persona de su mismo sexo, las diferencias fueron marginalmente significativas o no fueron significativas. En todos los casos, la experiencia de haber sido víctima de violencia se relacionó con mayor probabilidad de presentar ideación e intento de suicidio, consumo problemático de alcohol y consumo de drogas médicas. La violencia verbal y física en la familia y la violencia extrema en la escuela fueron las variables que más explicaron las diferencias en estos eventos de salud mental entre adolescentes con diferente orientación sexual.

Las actitudes hacia la homosexualidad fueron utilizadas como una aproximación a la experiencia del estigma asociado con la orientación sexual. La premisa fue que los adolescentes LBH que tuvieran actitudes negativas hacia la homosexualidad podrían experimentar homofobia internalizada; y por ende tendrían más probabilidad de presentar deterioro en su salud mental 17. Como era previsible, los adolescentes que tuvieron un noviazgo o relaciones sexuales con personas de su mismo sexo tuvieron mayor probabilidad de dar respuestas (en dos de tres preguntas) que denotan actitudes positivas o neutras hacia la homosexualidad; lo que denota que en ellos la homofobia, aunque existente, es menos prevalente.

$\mathrm{Al}$ analizar la relación de las actitudes hacia la homosexualidad con los eventos de salud mental, se observó que entre aquellos que dieron respuestas que denotan actitudes negativas hacia la homosexualidad tuvieron menor probabilidad de presentar síntomas de depresión, ideación e intento de suicidio y consumo problemático de alcohol. Este patrón deriva en gran medida de lo que ocurría en los adolescentes que no han tenido relaciones sexuales. Mientras que en los adolescentes que han tenido relaciones sexuales con personas de distinto sexo casi no existieron diferencias. En cambio, en los adolescentes que han tenido relaciones sexuales con personas del mismo sexo, tanto aquellos que tenían actitudes negativas, como los que tenían actitudes positivas tuvieron mayor riesgo de experimentar ideación e intento de suicidio (aunque en el último caso la diferencia fue marginal). Estos resultados muestran que el adherirse a los valores dominantes (i.e. la homofobia cultural) en la salud depende si se pertenece al grupo dominante o al grupo discriminado. En los adolescentes heterosexuales (i.e. el grupo dominante) el mantener una actitud negativa hacia la homosexualidad no tiene efecto o les confiere protección a su salud mental. Se ha planteado 18 que la homofobia en los heterosexuales tienen una función de protección de la autoestima al tener un grupo de referencia considerado inferior. En el caso de los adolescentes homosexuales (i.e. el grupo discriminado), aquellos que son portadores del estigma asociado a su sexualidad presentan peor salud mental, por lo menos en la conducta suicida. La mayor probabilidad de presentar síntomas de depresión y conducta suicida en los adolescentes homosexuales, que tienen actitudes positivas a la homosexualidad, es posible que se deba a que entre ellos se encuentran los que son más visibles y, por tanto, con más frecuencia han sido víctimas de violencia homofóbica.

Los resultados referentes a la actitud hacia a la homosexualidad también ilustran las dificultades para estudiar los efectos del estigma, utilizando diseños epidemiológicos convencionales. Es decir, los modelos de regresión se basan en la premisa de que las variables mediadoras aumentan el riesgo del evento, tanto en el grupo expuesto, como en el grupo de referencia; lo cual ocurrió con la violencia. Sin embargo, como ya se señaló, la relación de la actitud hacia la homosexualidad con los daños a la salud mental difiere, de acuerdo a si han tenido relaciones sexuales o no y el sexo de con quién han tenido relaciones. Es posible que la poca e inconsistente contribución de la actitud hacia la homosexualidad a las diferencias en morbilidad mental, de acuerdo con la orientación sexual se deba a esta limitación de la metodología utilizada.

En los adolescentes mexicanos entrevistados en la ENEIVEMS no existieron diferencias de acuerdo con la orientación sexual en un indicador positivo de salud mental (i.e. vitalidad), en la autoestima y en el consumo de tabaco. Lo anterior contrasta con el mayor riesgo de consumo de 
tabaco que se ha observado en países industrializados 19. Incluso en una muestra representativa de jóvenes mexicanos se observó 2 que aquellos que habían sentido atracción sexual o se autodenominaban como LBH tenían mayor probabilidad de fumar cigarros. Aunque es posible que las experiencias de discriminación asociadas con la orientación sexual no influyan en la autoestima; también es posible que estos resultados se atribuyan a las limitaciones de la encuesta analizada. Una de ellas tiene que ver con la medición de la orientación sexual. Las dos preguntas que fueron analizadas están relacionadas con el establecimiento de vínculos afectivos o sexuales con personas del mismo sexo. Sin embargo, las dimensiones afectivas y de atracción erótica de la orientación sexual no requieren de dichos vínculos para definir una orientación ${ }^{20}$. Es decir, un adolescente puede sentir atracción por personas de su mismo sexo y, sin embargo, no haber tenido relaciones sexuales o algún noviazgo con personas de su mismo sexo. Sin embargo, por el contenido de las preguntas él no será clasificado como homosexual. Además, en la población LBH el tener una relación de pareja con personas del mismo sexo aumenta la autoestima y reduce los niveles de homofobia internalizada 21 . Es decir, es posible que esté operando un sesgo de clasificación, el cual tenderá a producir estimaciones cercanas al valor nulo.

El sesgo de clasificación también puede verificarse al comparar las características sociodemográficas de los adolescentes de diferente orientación sexual. Los adolescentes que han tenido noviazgo, o relaciones sexuales con personas de su mismo sexo, tienden a ser de mayor edad, sus padres tienen mayor escolaridad y en ellos predominan los varones. El predomino de los varones puede atribuirse a que ellos inician antes su vida sexual. La diferencia en la edad puede ser resultado de que la homosexualidad sigue siendo estigmatizada, por lo cual los individuos tienen que elaborar una identidad a partir de su orientación sexual 22. Este proceso se realiza a diferentes edades en cada persona; pero sobretodo tiende a ocurrir al término de la adolescencia e inicio de la vida adulta. Por lo regular las personas con homofobia internalizada tienden a comenzar a edades posteriores la construcción de dicha identidad. Por otra parte, las personas de mayor escolaridad suelen presentar menos actitudes negativas hacia la homosexualidad 23; por lo cual es esperable que los adolescentes homosexuales cuyos padres tienen menos escolaridad sean más renuentes a aceptar y/o declarar su orientación sexual.

Otro sesgo que puede estar operando es el de selección. La población objetivo de la ENEIVEMS son los adolescentes inscritos en instituciones educativas, con lo cual se excluye a aquellos que no estudian. Los adolescentes LBH con mayor frecuencia se encuentran con dificultades en la escuela (tal como se observó con el análisis de la ENEIVEMS), por lo cual tienden a tener tasas más altas de deserción escolar 24. Por ello, probable que en la ENEIVEMS se haya excluido a algunos de los adolescentes LBH que se han enfrentado a actos de discriminación. De nuevo, este sesgo tenderá a oscurecer las diferencias en salud y experiencias de discriminación asociadas con la orientación sexual.

Como cualquier diseño transversal, la ENEIVEMS no permite distinguir la secuencia temporal en la que se presentaron la exposición, los mediadores y los eventos; por lo cual no es posible hacer conclusiones contundentes de causalidad y mediación. Además, aunque los resultados de los análisis de regresión son congruentes con el papel hipotético de la violencia como variable mediadora, dichos modelos en realidad no distinguen entre variables mediadoras y factores de confusión (especialmente cuando éstos producen supresión de efecto), ya que ambas variables pueden reducir o cancelar el efecto de la variables exposición 25 . Por otro lado, no se exploraron todas las formas de discriminación interpersonal, institucional o cultural. Por ejemplo, aunque las personas no hayan sido víctimas de un acto de violencia; el haber observado a personas LBH siendo víctimas de discriminación o el convivir con personas con actitudes negativas hacia la homosexualidad pueden ser estresores.

El trabajo de campo de la ENEIVEMS se realizó antes de la aprobación de matrimonio para personas del mismo sexo en la ciudad de México (2010) y en el estado de Coahuila (2014). En el futuro convendría analizar si los cambios institucionales y legales para proteger los derechos de las poblaciones LBH se reflejan en reducción de las disparidades en salud asociadas con la orientación sexual. Para ello se requiere la indagación sistemática de la orientación sexual en encuestas y censos, pues dicha información serviría para monitorear las desigualdades sociales y en salud relacionadas con la discriminación por orientación sexual. En términos metodológicos se requiere utilizar mediciones de la orientación sexual que la consideren como un constructo multi-dimensional y cambiante con el tiempo 20. Además, se ha sugerido que la orientación sexual debe ser considerada un continuo, por lo cual es más conveniente preguntar la identidad o las prácticas sexuales utilizando escalas tipo Likert.

En síntesis, los resultados del análisis aquí presentado muestran que, a pesar de los avances 
institucionales y legales para el reconocimiento de los derechos de la población LBH, persisten inequidades en salud relacionadas con la discri- minación por orientación sexual. Estas inequidades son verificadas incluso cuando es probable que operen sesgos que las pueden oscurecer.

\section{Resumo}

O objetivo deste estudo foi avaliar disparidades em saúde mental relacionadas com a discriminação baseada na orientação sexual em adolescentes do México. Estudou-se uma amostra nacional e representativa dos alunos do ensino médio. Foram utilizados dois indicadores de orientação homossexual: ter um namorado do mesmo sexo e tendo relações sexuais com alguém do mesmo sexo. Os eventos foram: depressão, autoestima, ideação suicida, e tentativa de suicídio e consumo de cigarros, álcool e outras drogas. Adolescentes que tinham compromissos ou relações sexuais com pessoas do mesmo sexo tiveram risco aumentado de sintomas depressivos, ideação suicida, tentativa de suicídio e consumo problemático de álcool. Essas diferenças foram relacionadas com a experiência da violência na família e na escola. Apesar dos desenvolvimentos institucionais e legais para o reconhecimento dos direitos da população lésbica, bissexual e homossexual, persistem desigualdades na saúde relacionadas com a discriminação por orientação sexual.

Homofobia; Saúde Mental; Comportamento Sexual; Violência

\section{Colaboradores}

L. Ortiz-Hernández contribuyó con la concepción del proyecto, análisis e interpretación de los datos, redacción del artículo, aprobación final de la versión para publicación y fue responsable de todos los aspectos del trabajo, incluyendo la exactitud e integridad de toda la obra. R. G. Valencia-Valero contribuyó con el análisis e interpretación de los datos, revisión crítica relevante del contenido intelectual del trabajo original, aprobación final de la versión que podría ser publicada, así como fue responsable de todos los aspectos del trabajo, incluyendo exactitud e integridad de toda la obra. 


\section{Referencias}

1. Commission on Social Determinants of Health. Closing the gap in a generation: health equity through action on the social determinants of health. Geneva: World Health Organization; 2008.

2. Ortiz-Hernández L, Tello BL, Valdes J. The association of sexual orientation with self-rated health, and cigarette and alcohol use in Mexican adolescents and youths. Soc Sci Med 2009; 69:85-93.

3. Herek GM, Garnets LD. Sexual orientation and mental health. Annu Rev Clin Psychol 2007; 3: 353-75.

4. King M, Semlyen J, Tai SS, Killaspy H, Osborn D, Popelyuk D, et al. A systematic review of mental disorder, suicide, and deliberate self harm in lesbian, gay and bisexual people. BMC Psychiatr 2008; 8:70.

5. Goffman E. Estigma. La identidad deteriorada. México DF: Amorrortu Editores; 1998.

6. Meyer IH. Minority stress and mental health in gay men. J Health Soc Behav 1995; 36:38-56.

7. González-Forteza C, Wagner FA, Jiménez A. Escala de Depresión del Centro de Estudios Epidemiológicos (CES-D) en México: análisis bibliométrico. Salud Mental 2012; 35:13-20.

8. Musitu G, García F, Gutiérrez M. AFA: autoconcepto forma-A. 2a Ed. Madrid: TEA Ediciones; 1994.

9. Borges G, Orozco R, Medina Mora ME. Risk index for attempted suicide in Mexico. Salud Pública Mex 2012; 54:595-606.

10. Global Youth Tobacco Survey Collaborative Group. Tobacco use among youth: a cross country comparison. Tobacco Control 2002; 11:252-70.

11. Courtney KE, Polich J. Binge drinking in young adults: data, definitions, and determinants. Psychol Bull 2009; 135:142-56.

12. Fillmore MT, Jude R. Defining "binge" drinking as five drinks per occasion or drinking to a $.08 \% \mathrm{BAC}$ : which is more sensitive to risk? Am J Addict 2011; 20:468-75.

13. Karlson KB, Holm A, Breen R. Comparing regression coefficients between models using logit and probit: a new method. Sociol Methodol 2012; 42:286-313.
14. Karlson KB, Holm A. Decomposing primary and secondary effects: a new decomposition method. Res Soc Stratif Mobil 2011; 29:221-37.

15. Consejo Nacional para Prevenir la Discriminación. Encuesta Nacional Sobre Discriminación en México 2010. México DF: Consejo Nacional para Prevenir la Discriminación; 2011.

16. Katz-Wise SL, Hyde JS. Victimization experiences of lesbian, gay, and bisexual individuals: a metaanalysis. J Sex Res 2012; 49:142-67.

17. Ortiz Hernández L. Influencia de la opresión internalizada sobre la salud mental de bisexuales, lesbianas y homosexuales de la ciudad de México. Salud Mental 2005; 28:49-65.

18. Herek GM. Beyond "homophobia": a social psychological perspective on attitudes toward lesbians and gay men. J Homosex 1984; 10:1-21.

19. Needham BL. Sexual attraction and trajectories of mental health and substance use during the transition from adolescence to adulthood. J Youth Adolesc 2012; 41:179-90.

20. Sell RL. Defining and measuring sexual orientation: a review. Arch Sex Behav 1997; 26:643-58.

21. Bauermeister JA, Johns MM, Sandfort TG, Eisenberg A, Grossman AH, D’Augelli AR. Relationship trajectories and psychological well-being among sexual minority youth. J Youth Adolesc 2010; 39:1148-63.

22. Rotheram-Borus MJ, Fernandez MI. Sexual orientation and developmental challenges experienced by gay and lesbian youths. Suicide Life Threat Behav 1995; 25 Suppl:26-34.

23. Herek GM, McLemore KA. Sexual prejudice. Annu Rev Psychol 2013; 64:309-33.

24. Russell ST, Seif H, Truong NL. School outcomes of sexual minority youth in the United States: evidence from a national study. J Adolesc 2001; 24:111-27.

25. MacKinnon DP, Krull JL, Lockwood CM. Equivalence of the mediation, confounding and suppression effect. Prev Sci 2000; 1:173-81.

Recibido el 28/Abr/2014

Versión final presentada el 02/Oct/2014

Aprobado el 08/Oct/2014 